\title{
A Study on the Novel Rectangular Split Ring Notch Resonators
}

\author{
Tangyao Xie, Girae Kim and Youngkyu Choi, Member, KIMICS
}

\begin{abstract}
In this paper, a novel notch resonator is proposed based on Rectangular Split Ring Resonator (RRSS) element. We represented the electrical characteristics for R-SRR elements coupled with microstrip line, and measured result is compared with electromagnetic simulation (HFSS) result. We also solved equivalent circuit for R-SRR element, and also discussed influences of change structural parameters of R-SRR. The size of R-SRR is the most important parameters for frequency tuning. Also we can make tunable resonator form the basic structure. This novel resonator can apply to design of tunable bandpass filter and voltage control oscillator.
\end{abstract}

Index Terms- metamaterials; notch resonator; Rectangular Split Ring Resonator (R-SRR); tunable filter

\section{INTRODUCTION}

Resonator forms the basic design elements in many circuit components including filter, oscillator, couplers and antennas. Planar resonators offer several advantages over conventional rectangular/circular waveguide resonators including size, weight and cost. The microstrip resonator was introduced by Troughton [1] in 1968. Since its introduction, the microstrip resonator has been used widely.

Recently, metamaterials attracts increasing interest in the engineering and scientific communities. The metamaterials is an artificial structure which does not exist in nature. Therefore, it provides another method and concept to develop various kinds of microwave circuits and its application. As important part of metamaterials, split ring resonators (SRRs) have attracted many interests in recent years as key constituent particles for the design of effective media with negative magnetic permeability or left-handed metamaterials (LHM). Originally proposed by Pendry [2], SRRs are sub-wavelength resonators able to inhibit signal propagation in a narrow band in the vicinity of their resonant frequency, provided the magnetic field is polarized along the axis of the ring. Recent strong experimental efforts have been directed towards the attainment of metamaterials with negative response in the Terahertz [3] and even optical $[4,5]$ frequency domain.

\footnotetext{
Manuscript received May 24, 2010; revised June 5, 2010; accepted June 16, 2010.

Authors are with the Department of Electronic Engineering, Silla University, Busan, 617-736, Korea. (Email: xietangyao@hotmail.com)

*Girae Kim is Corresponding Author (Email: grkim@silla.ac.kr)
}

In this paper, we proposed a novel notch microstrip resonator based on Rectangular Split Ring Resonator (RRSS) element. A $50 \mathrm{Ohm}$ microstrip line is on the top with one R-SRR element. In this work, we essentially compared the measurement with electromagnetic simulations. The simulated performance was obtained using simulator HFSS (V10), and measurement is accomplished with Anritsu MS4644A network analyzer. The measured results and simulation result show the good characteristic. And we also discussed the different influence of different parameters. Numbers of R-SRR cell, the gap between microstrip line and R-SRR cell, and the size (including the width of cell) of the cell are discussed. Using HFSS, we can get the results for different parameters. The size of R-SRR (including the width of cell) is the most important parameters for frequency tuning. Resonant frequency is changed widely when the size of cell changed. This resonator we proposed can be used in oscillator and bandpass filter. Also we can make tunable resonator form the basic structure.

\section{R-SRR ELEMENT}

The basic cell of the structure is represented in Fig. 1. R-SRRs can be modeled as LC resonant tanks that can be externally driven by a magnetic field and are therefore able to inhibit signal propagation in a certain narrow band if they are properly oriented [6]. R-SRRs also exhibit cross-polarization effects (magneto-electric coupling). Since the equivalent capacitance is given by the edge capacitance between concentric rings, the resonant frequency can be made very small by decreasing the rings' separation(g). In recent papers [7-11], the equivalent circuit model of a basic cell of SRRs is shown in Fig. 2. As shown in Fig. 2, $L_{r}$ is effective inductances; $C_{L}$ is effective capacitance. The parameters affecting the characteristic of R-SRR cell include the shape, size, gap and so on.

We designed one sample structure to measure the characteristics of R-SRR element as shown in Fig. 3. The $50 \mathrm{ohm}$ microstrip line is electrically coupled with one RSRR cell. The substrate is Teflon with dielectric constant $\varepsilon_{\mathrm{r}}=2.3$, thickness $\mathrm{h}=31 \mathrm{mil}$ ). All dimensions of structure are $\mathrm{B}=50 \mathrm{~mm}, \mathrm{~s}=0.2 \mathrm{~mm}, \mathrm{D}=16 \mathrm{~mm}, \mathrm{w}=2.36 \mathrm{~mm}$, and $\mathrm{g}=\mathrm{c}=\mathrm{d}=1.5 \mathrm{~mm}$. The photograph of fabricated is shown in Fig. 4. The simulated characteristic was obtained using simulator HFSS (V10), and measurement is accomplished 
with Anritsu MS4644A network analyzer. The simulated and measured results are shown in Fig.5. Referring to the measured results, the resonant frequency is about $2.02 \mathrm{GHz}$. The insertion loss is mainly about $-15 \mathrm{~dB}$. Good agreement between the simulation and measurement is achieved.

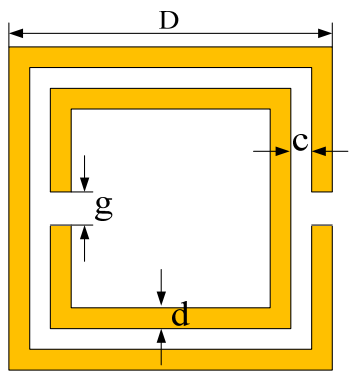

Fig.1 R-SRR element

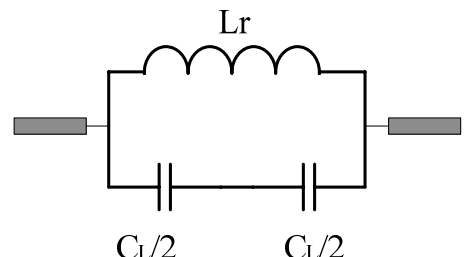

Fig. 2 Equivalent Model of R-SRR cell

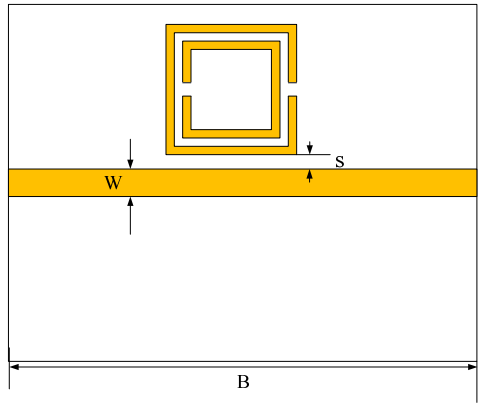

Fig. 3 Test Sample for R-SRR

$(\mathrm{B}=50, \mathrm{~s}=0.2, \mathrm{D}=16, \mathrm{~g}=\mathrm{c}=\mathrm{d}=1.5, \mathrm{w}=2.36 \mathrm{~mm})$

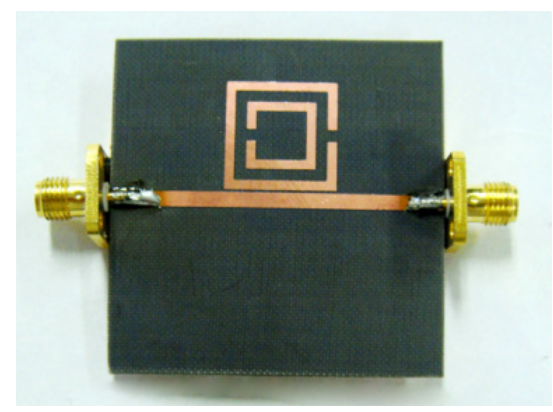

Fig. 4 Photograph of R-SRR with one cell

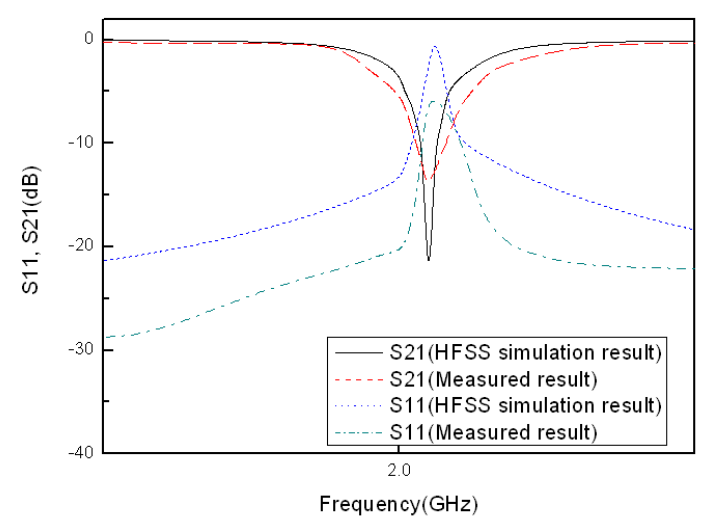

Fig. 5 Characteristics Results of R-SRR

\section{EQUIVALENT CIRCUIT FOR R-SRR ELEMENT}

The R-SRR resonator equivalent circuit model is depicted in Fig. 6. $C_{r}$ and $C_{L}$ are the edge capacitances corresponding to the equivalent of R-SRR cell; $\mathrm{L}_{\mathrm{r}}$ is inductance corresponding to the equivalent of R-SRR cell. Both couplings have been properly modeled by means of a mutual inductance $\mathrm{M}$ (magnetic coupling) and the edge capacitance between the line and the external ring $\mathrm{C}_{\text {edge }}$ (electric coupling). $\mathrm{C}_{\mathrm{rL}}$ and $\mathrm{C}_{\mathrm{rR}}$ are small. Except for the mutual inductance $\mathrm{M}$, the other element values have been estimated either through geometrical considerations or with the help of the commercial software Agilent ADS. The simplified equivalent circuit model of Fig. 6(a) can be further simplified to that shown in Fig. 6(b).

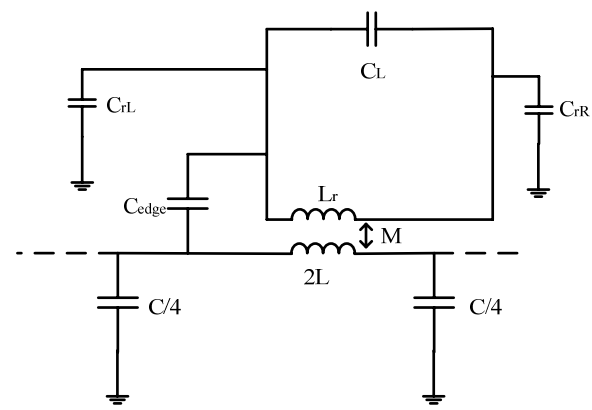

(a) Equivalent Model of R-SRR

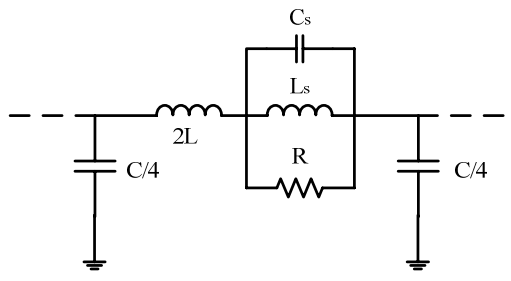

(b)Simplified circuit

Fig. 6 Equivalent circuit of R-SRR 
Using the equation (1) to (7), we can calculate the simplified equivalent circuit element values. The voltagecurrent equations for the transformer are first derived, i.e.,

$$
\begin{gathered}
V(j \omega)=j \omega 2 L I_{1}(j \omega)+j \omega M I_{2}(j \omega) \\
-I_{2}(j \omega) Z_{a}(j \omega)=j \omega 2 M I_{1}(j \omega)+j \omega L_{r} I_{2}(j \omega)
\end{gathered}
$$

where $Z_{a}$ is the impedance of $\mathrm{C}_{\mathrm{L}}$, namely,

$$
Z_{a}(j \omega)=\frac{1}{j \omega C_{L}}
$$

By isolating $I_{2}$ in (2) and substituting it into (1), we directly obtain the series impedance of the line

$$
Z_{S}(j \omega)=j 2 \omega L+\omega^{2} M^{2}\left\{\frac{j \omega C_{L}}{1-\omega^{2} L_{r} C_{L}}\right\}
$$

This impedance is equivalent to the series connection of an inductor (with inductance 2L) and a parallel RLC tank, provided the following conditions are satisfied:

$$
\begin{aligned}
& L_{s}=\omega_{0}^{2} M^{2} C_{L} \\
& C_{s}=L_{r} / \omega_{0}^{2} M^{2} \\
& R=\omega_{0}^{2} M^{2} / R_{s}
\end{aligned}
$$

We can get the element values from equations: $\mathrm{C}_{\mathrm{L}}=1 \mathrm{pF}$, $\mathrm{L}_{\mathrm{r}}=3.81 \mathrm{nH}, \quad \mathrm{C}_{\mathrm{rL}}=1 \mathrm{pF}, \quad \mathrm{C}_{\mathrm{rR}}=1 \mathrm{pF}, \quad \mathrm{L}=1.8975 \mathrm{nH}, \quad \mathrm{C}_{\text {edge }}=$ $0.792 \mathrm{pF}, \mathrm{C}=3.6 \mathrm{pF}$. The circuit simulation result using the ADS tool of Agilent is compared with others in Figure 7. It is agreed with measured and HFSS simulation result as shown in Figure 7.

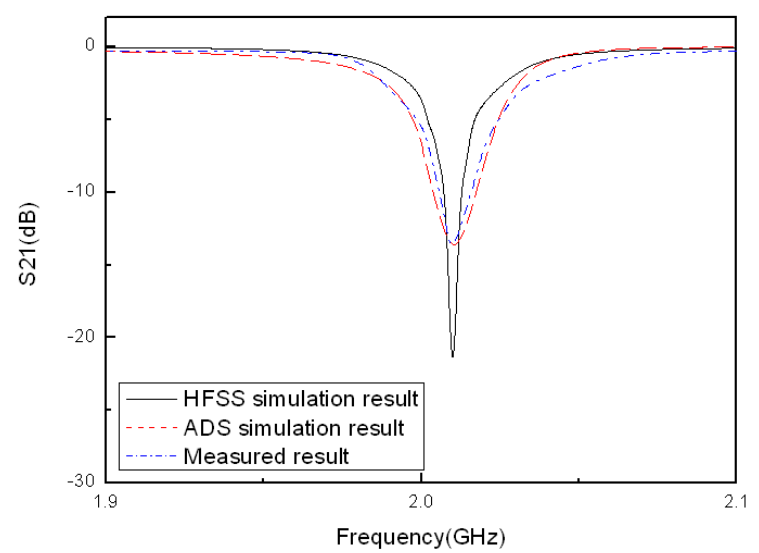

Fig. 7 Simulation Results and Measured Results
The insertion loss of a conventional bandstop resonator is given by:

$$
I L=10 \log \left[1+\frac{1+4 R}{4 R^{2}+4 Q_{u}^{2} R^{2}\left[\frac{\omega}{\omega_{0}}-\frac{\omega_{0}}{\omega}\right]}\right] \mathrm{dB}
$$

The 3-dB bandwidth is:

$$
\Delta_{3 \mathrm{~dB}}=\omega_{0} \sqrt{\frac{1+4 R-4 R^{2}}{4 Q_{u}^{2} R^{2}}}
$$

Now let $\frac{\omega_{0}}{\Delta_{3 d B}}=\frac{Q_{u}}{2}$ equal to (9)

$$
\frac{2}{Q_{u}}=\sqrt{\frac{1+4 R-4 R^{2}}{4 Q_{u}^{2} R^{2}}}
$$

Therefore at $\omega_{0}$ :

$$
I L=10 \log \left[1+\frac{1+4 R}{4 R^{2}}\right]
$$

\section{DISCUSSION for R-SRR CELL PARAMETERS}

In this section, we discuss some parameters of R-SRR cell to get the most important influence one for the notch resonator. First, we changed the numbers of R-SRR cell. In Fig. 8, we changed the cell number into two and four. All the dimensions are the same with previous proposed structure. We also use simulator HFSS to get the simulation results. The simulation results are shown in Fig. 9. The resonant frequencies for different cell number are nearly the same; frequency difference among them is about maximum $10 \mathrm{MHz}$. One cell resonant frequency is $2.03 \mathrm{GHz}$, resonant frequency for two cells is $2.023 \mathrm{GHz}$, and resonant frequency for four cells is $2.012 \mathrm{GHz}$. According to increase number of cell, there are coupling capacitances between cells, so the resonant frequency is move to lower a little bit. But, if the gap between cells is enough, we can ignore the influence of coupling capacitance between cells. According to the analysis of equivalent circuit, even if cell numbers are changed, capacitance $C_{L}$ and inductance $L_{r}$ have not changed. Due to the cell number, the S21 insertion loss has changed.

The second parameter we will discuss is the size D of cells between in Fig. 1. We choose the different size of D $(12 \mathrm{~cm}, 16 \mathrm{~cm}$ and $20 \mathrm{~cm})$. In Fig. 10, the simulation results are presented (use HFSS V10). The resonance 
frequencies are $2.02,1.5,1.3 \mathrm{GHz}$ for $\mathrm{D}=16,12,20 \mathrm{~mm}$. The insertion loss also has different. From the frequency, there is a big different value. And according to the equivalent circuit, we can conclude that there is big influence with the size.

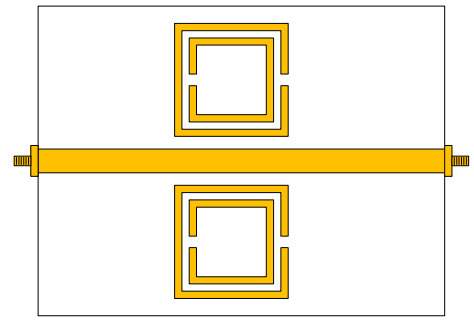

(a) Proposed R-SRR with two cells

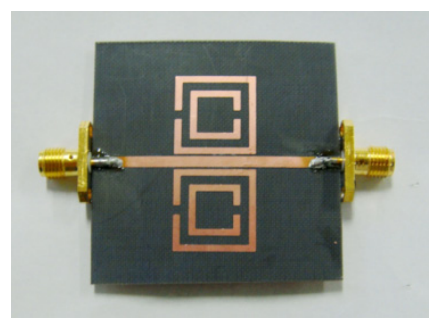

(b) Photograph of R-SRR with two cells

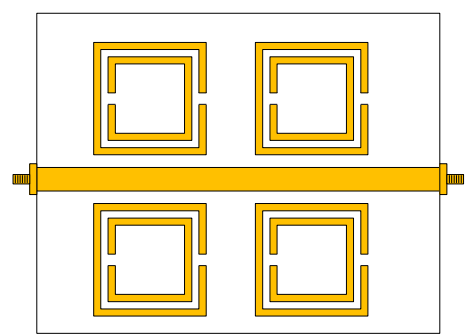

(c) Proposed R-SRR with four cells

Fig. 8 R-SRR Structures

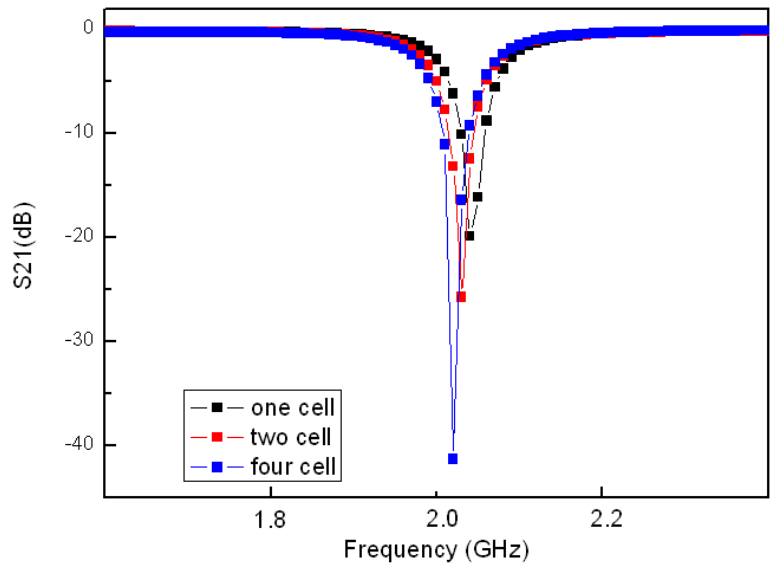

Fig. 9 The simulation results with different cell numbers

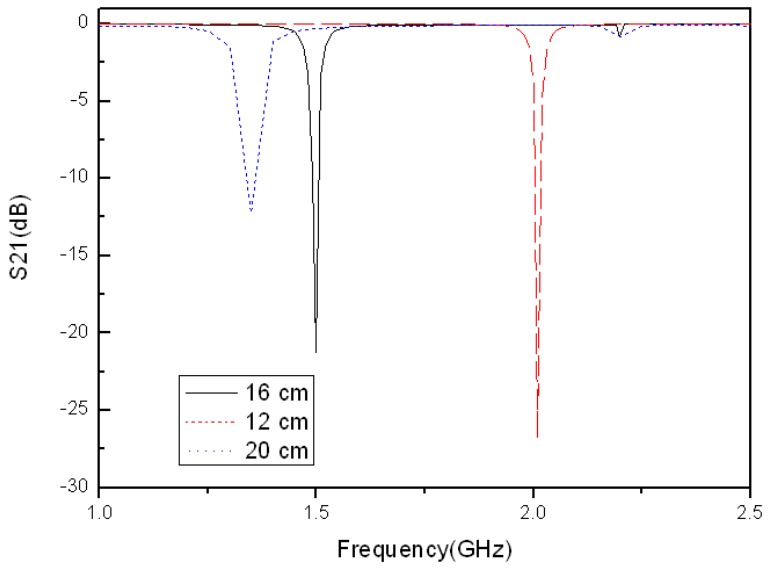

Fig. 10.The simulation results with different cell size

\section{CONCLUSIONS}

In summary, it has demonstrated that a compact notch filter has been presented, simulated and measured. The measured results and simulation result show the good characteristic. And we also discussed the different influence of different cell parameters. Numbers of R-SRR cell, and the size (including the width of cell) of the cell are discussed. Using HFSS V10, we can get the results for different parameters. The size of R-SRR (including the width of cell) is the most important parameters for frequency tuning. Resonant frequency is changed widely when the size of cell changed. This resonator we proposed can be used in oscillator and bandpass filter. Also we can make tunable resonator form the basic structure.

\section{REFERENCES}

[1] P. Troughton, "High Q-factor resonator in microstirp," Electro. Lett., vol. 4, pp. 520-522, 1968.

[2] J. B. Penry, A. J. Holden, D. J. Robbins, and W. J. Stewart, "Magnetism from conductors and enhanced nonlinear phenomena," IEEE Trans. Microw. Theory Tech., vol. 47, no.11, pp. 2075-2084, Nov. 1999.

[3] S. Linden, C. Enkrich, M. Wegener, J. Zhou, T. Koschny, and C. M. Soukoulis, "Magnetic response of metamaterials at 100terahertz", Science 306, pp. 1351-1353, 2004.

[4] C. Soukoulis, "Bending back light: The science of negative index materials," Optics and Photonics News, vol. 17, No. 6, pp. 18-21, 2006.

[5] V. M. Shalaev, W. Cai, U. K. Chettiar, H. K. Yuan, A. K. Sarychev, V. P. Drachev, and A. V. Kildishev, "Negative index of refraction in optical metamaterials," Opt. Lett. Vol.30, pp. 3356$3358,2005$.

[6] R. Marqués, F. Mesa, J. Martel, and F. Medina, "Comparative analysis of edge and broadside couple split ring resonators for metamaterial design. Theory and experiment", IEEE Trans. Antennas Propag., vol. 51, no. 10, pp. 2572-251, Oct. 2003.

[7] J. D. Baena, etc. "Equivalent-Circuit Models for Split-Ring Resonators and Complementary Split-Ring Resonators Coupled to Planar Transmission Lines", IEEE Trans. Microwave Theory \& Tech., vol. 53, no. 4, pp.1451-1461, 2005. 
[8] C. Cenk, A. Sondas, Y. E. Erdemli, "Tunable split ring resonator microstrip filter Design", Proc. Mediterranean Microwave Symposium, Genova, Italy, Sep., 2006.

[9] I. Gil, etc. "Tunable Metamatrial Transmission Lines Based on Varactor-Loaded Split-Ring Resonators", IEEE Trans. Microwave Theory \& Tech., vol. 54, no.6, pp.2665-2674, 2006.

[10] I. Gil, etc. "Varactor-loaded split ring resonator for tunable notch filters at microwave frequencies", Electro. Lett., vol. 40, no. 21, 2004.

[11] G.R. Kim, Tangyao Xie, "Frequency variable DGS type Resonator using Varactor Diode" Joint Conference, Korea Communication Society, Vol-18, No.2, pp.19-21,2009.

[12] G.R. Kim, "A study on Characteristics of Frequency Variable Resonator using Donut type DGS", Journal of Korea Electronic Information Communication Technology, Vol-2,No-4. pp.59-64, 2009.

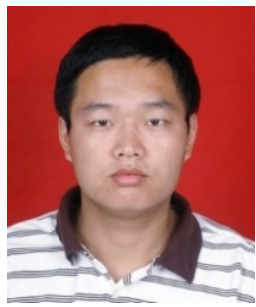

Tangyao Xie

received the B.S. degree in communication engineering from North China Institute of Science and Technology (NCIST), China in 2008. He is currently pursuing the M.S. degree in electronic engineering, Silla University, Busan, Korea. His research interests include microwave and RF circuit design.

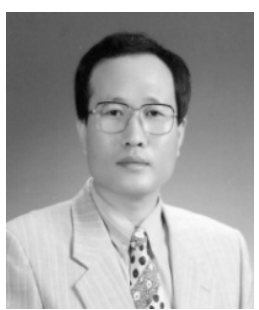

\section{Girae Kim}

received the B.S., M.S. in Electronic Engineering from the Sogang University, Seoul, Korea, in 1986, 1988, respectively. He also received Ph.D. from Kyungnam University, Korea in 1999. From 1988 to 1993 he was a researcher in Communication Research Center of Samsung Electronics Co. Ltd. Since 1999, he has been on the faculty of Electronics Engineering Department at the Silla University, Busan, Korea.

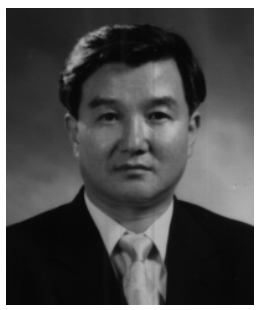

\section{Young-Kyu Choi}

received the B.S. in Electronic Engineering form the Chung-Ang University, Seoul, Korea, in 1982, and M.S. and Ph.D degrees from Kyoto University, Kyoto, Japan in 1989, and 1992, respectively. From 1992 to 1995 , he was a full-time lecturer of the Department of Electronics, Fukui University, Fukui, Japan. He is now an associate professor of the Department of Electronics, Silla University, Busan, Korea. 